\title{
Enabling personalized medicine in Europe: a look at the European Commission's funding activities in the field of personalized medicine research
}

The potential benefits that personalized medicine approaches can bring to healthcare are discussed with much interest throughout the EU. This novel approach is based on a better understanding of the molecular mechanisms of health and disease and it has now begun to show results. There is, however, a long way to go before the area is fully exploited. This special report reviews the European Commission's funding activities related to personalized medicine research. Although the specific focus on personalized medicine is relatively new, the European Commission has already committed significant funding to research in enabling technologies relevant to the field, as well as to specific disease areas for their application. A stocktaking exercise which started in $\mathbf{2 0 1 0}$ has helped identify immediate and future challenges in personalized medicine research. The outcome of this exercise, summarized below, will contribute to developing a vision for future research funding at the EU level.

\section{KEYWORDS: biomarkers diagnostics education and training enabling technologies EU Horizon 2020 personalized medicine pharmacogenomics rare diseases Seventh Framework Programme}

The goal of medicine has always been to provide the best possible care for patients. Personalized medicine promises to advance this goal further by employing a "medical model using molecular profiling technologies for tailoring the right therapeutic strategy for the right person at the right time, and determine the predisposition to disease and to deliver timely and stratified prevention" [101]. Major benefits can be expected for patients in terms of increased efficiency and less adverse reactions to prescribed treatments. Personalized medicine may also have positive impacts on healthcare costs. Although stratifying patients into subgroups, sharing the same molecular characteristics as a first step to personalized approaches, is already practiced in some therapeutic areas, such as cancer and HIV/AIDS, significant research and development efforts are needed across the full innovation chain in many other medical areas. This includes basic research through the clinical development phases to health economics.

\section{Enabling research at the European level}

To gain a better understanding of the key research needs for the development of personalized medicine approaches, the European Commission's (EC) Personalised Medicine Unit in the Health Research Directorate organized the conference European Perspectives in Personalised Medicine. The conference, held in
Brussels on 12-13 May 2011, attracted some 450 participants from across Europe and beyond.

The EC has committed significant funding over the last 5-year period (2007-2011) via the Health Theme of the Seventh EU Framework Programme for Research and Technological Development (FP7) [102] to research enabling the development of personalized medicine approaches. Collaborative projects harnessing talents from the EU and around the globe have received some $€ 900$ million in support of research and development in the following domains: tools and technologies for high-throughput research; new diagnostics development; and large-scale data gathering, in particular for 'omics research such as genomics, proteomics, metagenomics and epigenomics. Several EU funded collaborative projects aim at developing generic tools and knowledge that should impact directly on progress in personalized medicine for major disorders such as cardiovascular diseases, CNS diseases and cancer. Advances are expected in the identification of susceptibility genes to predict diseases, novel targets to overcome drug resistance and biomarkers to stratify patient populations in subgroups. Additional projects are specifically developing stratified or personalized approaches to breast, colorectal, renal, adrenal and lung cancer [103].

Other projects aim to structure and support European participation in large-scale international research collaborations. In these endeavors, where vast amounts of data are gathered

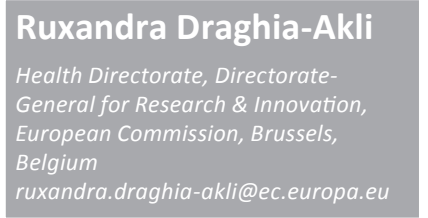

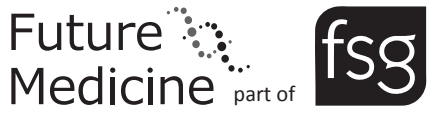


and structured, individually funded projects from several international and national funding agencies work together towards common goals. This type of collaboration ensures open and free access to data and resources, avoiding overlapping research funding. It is, moreover, instrumental when it comes to establishing internationally approved high-quality data standards.

Examples particularly relevant for the development of personalized medicine approaches include the International Cancer Genome Consortium (ICGC) [104] that aims to obtain a comprehensive description of genomic, transcriptomic and epigenomic changes in 50 different tumor types and/or subtypes, which are of clinical and societal importance across the globe; the International Human Epigenome Consortium (IHEC) [105], which coordinates the production of reference maps of at least 1000 human epigenomes for key cellular states relevant to health and disease; the International Knockout Mouse Consortium (IKMC) [106], which aims at mutating all protein-coding genes in mice in order to unravel the genetic functions underlying disease; and the International Human Microbiome Consortium (IHMC) [107], which explores the role of the human microbiome in the maintenance of health and causation of disease in order to improve the ability to prevent and treat disease. Based on such international collaboration, EU-funded researchers were able to publish the first ever human metagenome in March 2010 [1].

\section{Addressing future research needs}

To further understand the specific research needs where funding at the EU or international level would provide added value for the development of personalized medicine, a series of workshops were organized throughout 2010 culminating in the European Perspectives in Personalised Medicine conference [108]. The conference program covered the full innovation chain - from basic research, throughout the clinical development phases, the approval and postapproval processes, as well as the experiences of use in the healthcare setting and by patients. The aim of this approach was to ensure that key challenges were identified, not only from scientific and technical viewpoints, but that challenges concerning implementation in healthcare and experiences from clinicians and patients were also taken into account.

The conference, gathering the opinions of participants from academia, small and large industry, policy-makers and regulators confirmed that although important research is ongoing, substantial challenges need to be overcome in order to make progress in the development of personalized medicine approaches. These challenges have been grouped under four headings in an attempt to capture the most urgent and/or important research needs.

\section{Breaking barriers \& speaking the same language}

Stratified, individualized and personalized medicine will require an unprecedented level of cooperation along the healthcare innovation chain starting with researchers who characterize diseases and elucidate their mechanisms, to those who exploit this knowledge by developing new biomarkers, diagnostics and medicines, and to the regulators who evaluate and approve them. The chain then continues to include the health technology assessors who appraise the value of the treatment, the health authorities who decide on the possible reimbursement of its costs and the clinicians who will treat the patients. An unprecedented effort should be made to bring together stakeholders to discuss common objectives and paths to progress.

In addition, real collaboration between various scientific disciplines from basic to clinical research, including biology, mathematics, statistics, pathology and medicine is needed. Platforms bringing together scientists from different areas should be created or developed. This requires new and improved infrastructures, and interfaces for materials and information exchanges.

Substantial education and training efforts are needed to ensure that knowledge and good practice concerning novel technologies and scientific approaches are shared. Information on scientific breakthroughs, novel technologies and practices should also be provided in a language easily accessible to the public.

\section{Generating knowledge \& developing the right tools}

A key challenge in the path towards personalized medicine lies in the development of tools and technologies to enable the discovery and validation of new biomarkers, as these are increasingly used to stratify different patient groups in terms of clinical response and susceptibility to treatment. The vast amount of data generated by 'omics-technologies (e.g., genomics, proteomics, metagenomics and epigenomics) provides the potential to obtain a global view on the molecular and cellular processes in health and diseases. This has created a new challenge for data analysis and management. There is a need for internationally recognized standards regarding the collection 
and analysis of data, while new mathematical and statistical models and novel algorithms are needed to explore the complex molecular profiles obtained by analyzing the vast amount of data.

Furthermore, by studying known monogenic disorders, our understanding of genetic and environmental modifiers of disease can be substantially improved. This can provide a basis for the discovery, evaluation and validation of novel predictive biomarkers and molecular signatures that can be used for stratified and personalized therapies. The discovery of the genetic basis of the Mendelian forms of common diseases can result in novel insights into the molecular mechanisms of diseases and may also help to identify novel drug targets for common diseases.

\section{Translation to medical applications}

Biomarkers allow for the assessment of the response to therapeutic interventions in terms of safety and efficacy. Consequently, they are increasingly used in the development and the use of new treatments. They can also play a major role in optimizing the use of existing treatments by reducing the risk of adverse reactions and ensuring that treatments are prescribed specifically to patients for which it has been proven to be efficacious.

Robust data should support the qualification of a defined biomarker in drug development before its clinical validation. The rate by which biomarkers are currently discovered is by no means matched by the rate of biomarker qualification and validation. It is believed that the attrition rate of biomarker development is comparable to the attrition rate of drug development. Some structured activities have been initiated in Europe and in the USA that will improve and speed up the translation of biomarker discoveries into clinical applications such as the biomarker qualification process by the EMA and the US FDA as well as the US NCI early detection research network. More such coordinated efforts are needed.

As patient populations are stratified into smaller groups, adaptive clinical trial designs where data gathered during the trial can be used to adapt the trial design before its completion, possibly based on new statistical models and the use of centrally deposited clinical trial data could be explored. The latter will help reduce redundancies and cut some of the overall costs associated with logistics and the clinical research. Patients are to become partners in relevant discussions on clinical trial design, addressing issues such as ethics, patient inclusion, and obviously, clinical and patient benefits. Continued dialogue between scientists and regulators should be encouraged.

\section{Economic aspects}

The uptake of personalized medicine in healthcare will depend on the potential impact for patients and on the economic viability of such approaches in the healthcare setting. The French National Cancer Institute is a pioneering example of large-scale implementation of personalized medicine with molecular testing provided for all French cancer patients before decisions on appropriate treatments are done, and where the initial economic analysis of this scheme is positive. For example, the French National Cancer Institute spent $€ 1.7$ million on testing for EGFR biomarkers in 16,724 lung cancer patients. The tests showed that only 1724 patients ( $10 \%$ of the tested individuals) would respond to the available treatments (gefitinib or erlotinib). The savings for not treating the 15,000 nonresponding patients amounted to $€ 69$ million based on the median treatment period of 8 weeks [109,110]. The economic savings should be paired with the savings in time and additional suffering for the close to $90 \%$ of tested patients who would not benefit from the treatments. As the organization of the European health systems differs from country to country, other examples of national, regional and/or local implementation are needed, for cancer, as well as for other therapeutic areas, to obtain a comprehensive view on the impact on health budgets.

Other aspects that need to be addressed concern the methodologies used for health technology assessment (HTA) in personalized medicine, where the treatment decisions are based on the results from molecular profiling, and methodologies for comparative cost-effectiveness and other pharmacoeconomic evaluations such as cost:benefit ratio, cost efficiency and cost utility.

A list of urgent challenges where action is needed is provided in the list at the end of this special report.

\section{Future perspective}

The Health Research Theme of FP7 has already started to address some of the challenges identified above with projects funded in 2011 specifically targeting personalized medicine approaches. These projects focus on the following: pharmacogenomic biomarkers for already established treatments in cancers; neurological and psychiatric disorders; development of human epigenomic profiles in hematopoietic cells; and last but not least, further technology development for personalized medicine applications. 
Some $€ 160$ million have also been earmarked for 2012 to support the development of technologies for patient group stratification, improved statistical methods for 'omics data and validation of biomarkers as well as novel methods for HTA. A specific focus has been given to rare diseases as models for personalized medicine via the systematic application of 'omics to groups of rare diseases, and the build-up of clinical bioinformatics linking the identified molecular profiles with current clinical descriptions. This particular effort will contribute to the recently established International Rare Diseases Research Consortium (IRDiRC) for which the EC has taken the lead together with other research funders across the globe to stimulate research and development for better diagnosis and treatment of rare diseases [111].

Further challenges to foster the development of personalized medicine will be pursued by the Health Research Theme in 2013. Priorities and budgets for the next 7-year EU funding program for research and innovation, Horizon 2020, was launched for discussion by the EU member states late 2011.

Some of the key challenges that need to be addressed include:

- Breaking barriers and speaking the same language:

- Facilitate collaboration between different disciplines, from basic to clinical research, including biology, mathematics, statistics, pathology and medicine;

- Creating interfaces for collaboration and discussions among stakeholders including: basic researchers, pathologists, clinicians, patients, industry, regulators and payers;

- Rapid integration of knowledge regarding relevant novel technologies and new scientific approaches in education and training curricula.

- Generating knowledge and developing the right tools:

- Adaptations of many research tools for use in clinical settings;

- Standards for data and sample collection and for analytical procedures together with harmonized, comprehensive coding systems, as well as novel statistical methods and algorithms for analysis of large volumes of complex and heterogeneous datasets;

- Linking clinical data with molecular profiles (clinical bioinformatics) and development of novel algorithms to explore the complex signatures obtained from 'omics platforms that in the long term can lead to molecular definition of disease in addition to refining or replacing the clinical definition;

- Translation of 'omics research into clinical applications, starting with rare (monogenic) diseases, and in population cohorts that are gender and age stratified and with a core set of phenotype info on health status including lifestyle data, diets and exposures to environmental and other factors.

- Translation to medical applications:

- Development of new approaches and methodologies for identification and validation of biomarkers;

- Identification, qualification and clinical validation of all types of biomarkers (including imaging biomarkers);

- Use of biomarkers for better use of existing therapies;

- Adapted clinical trial methodologies for stratified and/or small populations.

- Economic aspects:

- Studies on impact for patients and on the economic viability of personalized approaches in healthcare systems;

- Methodologies for HTA and HTAs of personalized medicine approaches taking into account the therapy as well as the predictive/ diagnostic tools;

- Comparative cost-effectiveness and other pharmacoeconomic studies on personalized medicine approaches.

\section{Conclusion}

Collaborative research funded by the EU research programs is a useful tool to address some of the issues identified above. European projects bring together a variety of different disciplines and they require collaboration between scientists from different European countries and welcome collaboration with research groups across the globe. These projects can also address the need for multidisciplinary research by bringing together academia, industry (including small- and medium-sized enterprises) and patient advocacy groups to address ambitious goals that would not be possible for one single group or country. Being a public funding body, the EC can fund research where private investors would have little interest, but where results could bring major benefits to patients or to the society as a whole. 


\section{Executive summary}

- Personalized medicine is a novel approach to healthcare based on a better molecular understanding of health and disease. Some personalized medicine approaches have begun to show results but much research is still needed to progress the area.

\section{Enabling research at European level}

- The European Commission (EC) has allocated some $€ 900$ million to personalized medicine, enabling research over the latest 5-year period via the Health Theme of the Seventh EU Framework Programme for Research and Technological Development (FP7).

- To gain a better understanding of key research needs in the area, the Personalised Medicine Unit of the ECs Health Research Directorate organized several workshops and a large-scale conference during the period between 2010-2011.

\section{Addressing future research needs}

- The key research challenges identified can be grouped around four main themes:

- Breaking barriers and speaking the same language: facilitating interaction between different disciplines from basic to clinical research by creating appropriate interfaces for collaboration and discussion among stakeholders.

- Generating knowledge and developing the right tools: adapting research tools to clinical use by developing common standards for data collection and linking clinical data with molecular profiles, for example, translating 'omics research into clinical application.

- Translation to medical applications: finding new approaches for identification, qualification and clinical validation of all types of biomarkers; improving the use of biomarkers for better use of existing therapies and adaptive clinical trial methodologies.

- Economic aspects: proving the economic viability and positive patient benefits of personalized medicine and developing methodologies for health technology assessments and for comparative cost-effectiveness studies on personalized medicine approaches.

\section{Future perspective}

- The EC will continue to invest in research fostering the development of personalized medicine approaches, both through its current FP7 Health Research Theme in 2012 and 2013 as well as in its next funding program for research and innovation - Horizon 2020.

\section{Disclaimer}

This article was written by R Draghia-Akli, Director of the Health Directorate, Directorate-General for Research and Innovation, European Commission (C) European Union, 2012. The views expressed in this article are those of the author and do not necessarily reflect the officialposition of the European Commission.

\section{Financial \& competing interests disclosure}

$R$ Draghia-Akli is the Director of the Health Directorate, Directorate-General for Research and Innovation, Europeam Commission. The author is the inventor of many patents and patent applications, which are linked to DNA technologies. The author has no other relevant affiliations or financial involvement with any organization or entity with a financial interest in or financial conflict with the subject matter or materials discussed in the manuscript apart from those disclosed.

No writing assistance was utilized in the production of this manuscript.

\section{References}

Papers of special note have been highlighted as: - of interest

1 Quin J, Ruiqiang L, Jeroen R et al. A human gut microbial gene catalogue established by metagenomic sequencing. Nature 464, 59-65 (2010).

\section{Websites}

101 Omics in personalised medicine, summary workshop report.

http://ec.europa.eu/research/health/pdf/ summary-report-omics-for-personalisedmedicine-workshop_en.pdf

102 Seventh Framework Programme for Research and Technological Development.

http://cordis.europa.eu/fp7/home_en.html

- $\quad$ FP7 is the short name for the Seventh Framework Programme for Research and Technological Development. This is the EU's main instrument for funding research in Europe and it will run from 2007 to 2013. FP7 is also designed to respond to Europe's employment needs, competitiveness and quality of life. Read more about FP7 on the website.

103 EU funded health research. http://ec.europa.eu/research/health/ index_en.html

104 International Cancer Genome Consortium. www.icgc.org

105 International Human Epigenome Consortium. www.ihec-epigenomes.org
106 International Knockout Mouse Consortium. www.knockoutmouse.org

107 International Human Microbiome Consortium. www.human-microbiome.org

108 Summary reports from the conference and workshops organised by the Personalised Medicine Unit.

http://ec.europa.eu/research/health/ policy-issues-pm-conferences-workshops_ en.html

109 French National Cancer Institute. www.e-cancer.fr/en

110 Key data presented by Fabien Calvo. http://ec.europa.eu/research/health/pdf/ event06/13052011/fabien-calvo_en.pdf

111 International Rare Diseases Research Consortium.

http://ec.europa.eu/research/health/ medical-research/rare-diseases/irdirc_en. html

- To date, funding organizations from the following countries have expressed their formal intent to join International Rare Diseases Research Consortium: Australia, Canada, Italy, France, Germany, the Netherlands, Spain, the UK and USA. 